\title{
Chemical Abundances of the Planetary Nebulae NGC 2392 and NGC 3242
}

\author{
C.H. Wu ${ }^{1}$, J.Z. Li ${ }^{1,2}$, Z.W. Chang ${ }^{1}$, C.Y. $\mathrm{Lin}^{1}$, J.Y. $\mathrm{Hu}^{2}$ and W.H. $\mathrm{Ip}^{1}$ \\ 1. Institute of Astronomy, National Central University, Chung-Li \\ 32054,Taiwan(Email: m879008@astro.ncu.edu.tw) \\ 2. Beijing Astronomical Observatory, National Astronomical \\ Observatory, Chinese Academy of Sciences, Beijing 100012, China
}

\begin{abstract}
Planetary nebulae represent the end product of the evolution of low mass stars with $M<8 M_{\odot}$. The central stars have masses between 0.55 and $0.85 M_{\odot}$. This means a large amount of material must have been distributed in the interiors of the PNe. The observed variations of the chemical compositions and mass distributions therefore carry important information about the nature of the associated AGB superwinds and Red Giant outflows.(Kwok et al,1978) A program for comprehensive multiwavelength study of $\mathrm{PNe}$ has been initiated at NCU. One recent study has to do with the spectrographic observations of the planetary nebulae NGC 2392 and NGC 3242 using the $2.16 \mathrm{~m}$ telescope of the Beijing Astronomical Observatory. Some preliminary results are presented here.
\end{abstract}

\section{NGC 2392 (The Eskimo Nebula) and NGC 3242}

The Eskimo Nebula (NGC 2392) is unique in the sense that its elliptical structure is infiltrated by filamentary structures reminiscent of the cometary knots seen also in the Helix Nebula. There is also the interesting question on the ionization structures and dynamical histories of its different layers. It is therefore important to obtain spectra with high spatial resolution covering key features. The bipolar nebula NGC 3242 has prominent brightness structures at its two ansas (Balick et al. 1993). The origin of these so-called Fast Low Ionization Emission Regions (FLIERs) is still unclear.

\section{Preliminary Results}

The observations were performed with the $2.16 \mathrm{~m}$ telescope of the Beijing Astronomical Observatory on Dec 24 2000. The slit is about 4' long and 2.5" wide. The spectral resolution is $200 \AA / 6 \mathrm{~mm}$, see Figure 1 . We are particularly interested in NGC 2392 because in a previous study by Wu (2000) the filamentary structures surrounding the central region were found to display strong symmetry. This effect could not be explained by the occurrence of Rayleigh-Taylor instability as proposed previously by Capriotti (1973). It is more likely that pairs of polar jets were responsible for such structures. Phillips and Cuesta 


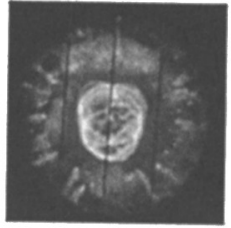

$\mathrm{NGC} 2392$

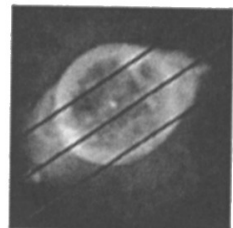

NGC 3242

Figure 1. Slit positions and orientations for the BAO observation of NGC2392 and NGC3242.

(1999) made similar observations in their analysis of their narrowband images of NGC 2392 in [O III] and [SII]. The whirling features in the central nebula are also puzzling. It might be that the wind-brown bubble mode for NGC 6543 (see Balick and Preston, 1987) could be applied to the Eskimo Nebula. We hope to use the present spectrographic data to shed further light on this issue.
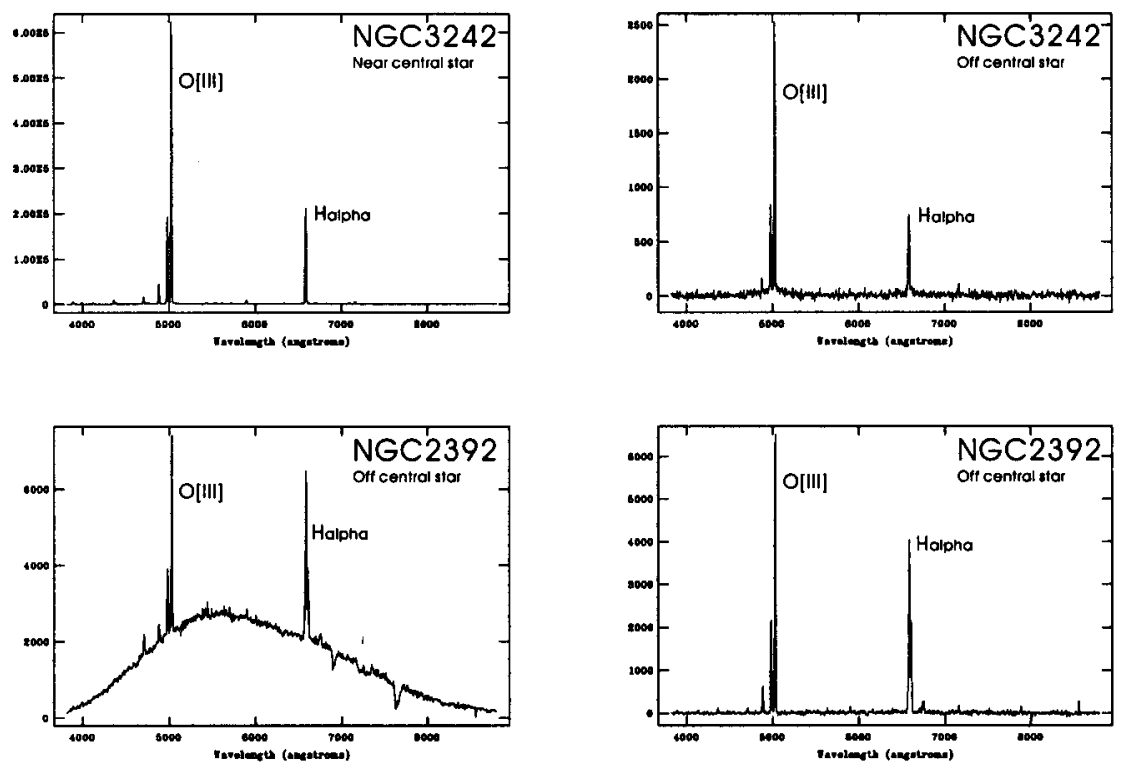

Figure 2. Preliminary spectrographic of NGC3242 and NGC2392. Left: spectrum taken near the central star. Right: spectrum taken off the central star.

\section{References}

Balick, B. and Preston, H.L. 1987, AJ, 94, 958

Balick B., M. Rugers, Terzian Y., Chengalur J.N. 1993, ApJ 411,778

Capriotti, E.R. 1973, ApJ 179, 495

Kwok S., Purton C.R., Fitzgerald P.M. 1978, ApJ 219, L125

Phillips, J.P. and Cuesta, L. 1999, AJ 118, 2929 